\title{
QOS MANAGEMENT IN AUTONOMIC HOME NETWORKS
}

\author{
Kaouthar Sethom, Nassim Laga, Guy Pujolle \\ LIP6, university of Paris 6, France \\ Firstname.lastname@lip6.fr
}

\begin{abstract}
Ambient intelligence is the driving force of the new digital world. The trend is to equip everyday life objects even inside home environment with intelligence, which will make our lives easier and more productive. Wireless mesh networking (WMN) has emerged as a promising concept to meet such challenges. This paper considers the problem of achieving QoS inside home networks relying on a wireless mesh networks.
\end{abstract}

Keywords: Home networking, mesh networks, routing

\section{INTRODUCTION}

Wireless mesh networks (WMNs) have emerged as a key technology for next-generation wireless networking. Because of their advantages over other wireless networks, WMNs are undergoing rapid progress and inspiring numerous applications. One example of such applications is "home networking". WMN is a self configuring and a self organizing network, which makes it a very suitable option for home networks. Communication within home can then be realized through mesh networking without going back to the access hub all the time. Thus, network congestion due to backhaul access can be avoided. Wireless mesh networking in the home could link desktop PCs, notebook and handheld computers, High-Definition TVs, DVD players, game consoles, 
camcorders, and other consumer electronic devices, without the need to pull cables, install network jacks or configure devices.

Traffics generated by this variety of applications, ranging from Internet browsing, data backup, and telephony, to entertainment and gaming have different requirements. A new Qos based routing protocol is thus needed for best management inside home.

Despite the availability of several routing protocols for ad hoc networks, the design of routing protocols for WMNs is still an active research area. In this paper, we present a novel routing protocol, named Wireless Mesh Protocol for QoS routing (WMP_QoS) inside home networks. The rest of the paper is organized in the following way. An analysis of routing metrics in mesh networks is presented in Section 2. In Section 3 we introduce our WMP_QoS protocol. Simulation results obtained using NS-2 are presented in Section 4. Section 5 concludes the paper.

\section{ROUTING IN WIRELESS MESH NETWORKS}

Wireless mesh networks (WMN) seem to be the best architecture for home networks for the following reasons:

- In a home, we can find nodes with high mobility (such as laptops, cameras, and mobile phones), and low mobility (such as PCs, and TV).

- The user is not an expert in the domain, so auto-configuration and auto-organisation are required functionalities. WMN have these properties.

- The components must be connected to the internet, so a pure adhoc network is not an optimal architecture.

Since WMNs share common features with ad hoc networks, the routing protocols developed for ad hoc networks were applied to WMNs. For example, Firetide Networks [1] are based on the TBRPF protocol [2], Another example is dynamic source routing (DSR) [3,4]: This protocol is divided into two parts: route discovery and route management. The first part discovers a path between a source and a destination, whereas the second one manages this route and notifies the source if there was a problem in the path (link failure). Source routing makes the protocol much more reactive and prevents loops. Moreover, the IEEE 802.11s standard [5] for wireless mesh networks defines a default mandatory routing 
protocol (Hybrid Wireless Mesh Protocol, or HWMP) that is inspired by a combination of AODV [6] and tree-based routing. However, Ad Hoc Networks like AODV and DSR decides the route on the basis of number of hops. However it is been proved by Woo et al.[7] that these minimum hop count paths degrades the performance of the network.

Selecting a good path is considerably harder in wireless networks than in traditional wired networks (where the routing problem is usually solved by running a distributed shortest-path algorithm on a graph) because the notion of a "link" between nodes is not well-defined. The properties of the radio channel between any pair of nodes vary with time, and radio communication range is often unpredictable. The communication quality of a radio channel depends on background noise, obstacles, and channel fading, as well as on other transmissions occurring simultaneously in the network.

To ensure good performance, routing metrics must satisfy four requirements. First, the routing metrics must not cause frequent route changes to ensure the stability of the network. Second, the routing metrics must capture the characteristics of mesh networks to ensure that minimum weight paths have good performance. Third, the routing metrics must ensure that minimum weight paths can be found by efficient algorithms with polynomial complexity. Finally, the routing metrics must ensure that forwarding loops are not formed by routing protocols.

There are some promising approaches for improving routing in wireless mesh networks. In this section, we will analyze the performance of four existing routing metrics for mesh networks: RTT [8], ETX [9], ETT [8], WCETT [10].

\subsection{Per-hop Round Trip Time (RTT)}

This metric is based on RTT measurement between a node and its neighbours. It was introduced by Adya and Al [3]. It is computed as follow:

- Every node sends a prob packet every $500 \mathrm{~ms}$; this packet contains the send time.

-Every node which receives this packet sends an acknowledgement which contains the send time retrieved in the received packet. This allows the source to compute the RTT.

-To avoid oscillations, the sending node keeps an exponentially weighted moving average of the RTT samples to each of its neighbours. 


\subsection{Expected Transmission Count (ETX)}

ETX is defined as the expected number of MAC layer transmissions that is needed for successfully delivering a packet through a wireless link. The drawbacks of ETX is that it does not consider interference or the fact that different links may have different transmission rates.

\subsection{Expected Transmission Time (ETT)}

This metrics traduces the number of required MAC retransmissions to reach a destination. To compute ETX, the implemented method works under 802.11 environment. This method will not work under other environment since it is based on the fact that 802.11 does not retransmit broadcast packets. To compute ETX we:

-Send probe packets every second to all neighbours.

-Every 10 seconds, report the number of received packet to the sending node.

The ETT metric captures the impact of link capacity on the performance of the path. However, the remaining drawback of ETT is that it still does not fully capture the intra-flow and inter-flow interference in the network [11].

\subsection{Weighted Cumulative ETT (WCETT)}

A routing protocol named (MR-LQSR) is proposed in [11] for multi-radio WMNs. A new performance metric, called the weighted cumulative expected transmission time (WCETT) is proposed for the routing protocol. WCETT have fixed some constraints:

-The routing protocol metric must reflect the loss rate and the bandwidth of the path.

-The metric must be increasing according to the path length.

-The metric must care of interference and the channel diversity.

\section{THE WMP_QOS PROTOCOL}

The goal of WMP_QoS is to build a WMN routing protocol that provides QoS guarantees to applications. This means that the service level and the network level cannot work as separated universes, each towards its own 
goals. Rather, the routes discovered by our routing protocol will feet to application requests for desired bandwidth and delay bounds for the flow, or deliver an end-to-end flow that satisfies those performance bounds at the time of the request. If and when the route is disrupted by node or link failure, the protocol automatically detects the route breakages, and rediscovers alternate routes if they exist. WMP_QoS is a reactive protocol that discovers routes on-demand.

\subsection{Service classes and QoS algorithm}

The objective of WMP_QoS is selecting network paths that have sufficient resources to satisfy the QoS requirements of the admitted connections. Many paths between the source and the destination may be available. Because there is no available centralized controller that knows the whole picture of the network resources, WMP_QoS calculates link weights hop by hop, and then combines them into a path metric. WMP_QoS is a source-routed protocol derived from AODV. Route discovery and metric calculation is based on Route request and route response.

\subsubsection{Assumptions}

We begin by listing the assumptions we made about the networks in which WMP_QoS is supposed to operate. These assumptions are not necessary for the correct operation of our protocol; they only simplify the case study. First, we suppose that the home network is only composed by three technologies: WiFi, Bluetooth and Ethernet. We assume that each service flow will provide the following QoS parameters: the minimum required bandwidth $B_{\min }$, the maximum end-to-end delay from the source to the destination, $T_{\max }$ and the minimum required security level $S_{\text {level. }}$.

Instead of shortest-path, WMP_QoS uses a combination of WCETT, available bandwidth $B_{\text {avai }}$, end-to-end delay $T_{\max }$, link energy $E_{i}$ and link security level $S_{i}$ as metrics.

The conceptual architecture of autonomic communications requires a knowledge plane to facilitate effective, transparent and high level selfmanagement capabilities. We assume that each node can get its available bandwidth $\mathrm{B}_{\text {avai }}$ and WCETTi on the current link $\mathrm{i}$ by simply asking the knowledge plane. 


\subsubsection{Route selection algorithm}

Our routing algorithm is implemented in the following four-step ondemand hop-by-hop route discovery procedure:

Step 1: Route Exploration: Nodes along possible routes are explored by the route request packets from the source. These packets travel through each node along the candidate routes to obtain bandwidth availability, link energy $E_{i}$ and link security level $S_{i}$ as well as gather the end-to-end delay information of the route.

Step 2: Route Registration: Bandwidth $B_{\min }$ is registered at each node along the reverse routes explored, by the route reply packets from the destination.

Path security level and Enery consumption on a particular route are then obtained from (1) and (2):

$\mathrm{S}=\min \mathrm{Si}$

$\mathrm{E}=$ sum $\mathrm{Ei} /$ number of hops

Step 3: Route selection:

- Case : application = voice

$\mathrm{P}=\left\{\text { Paths } / \text { delay }=<\mathrm{T}_{\max } \& \text { Security } \mathrm{S}>=\mathrm{S}_{\text {level }}\right\}_{\min \mathbf{E}}$

- Case $:$ application $=$ client $/$ server $($ email, telnet $\ldots)$

$P=\left\{\text { Paths } / S>=S_{\text {level }}\right\}_{\min }($ WCETT, $E)$

- Case $:$ application =file transfer

Path $=\left\{\text { Paths } / B_{\text {avai }}>=B_{\min } \text { and } S>=S_{\text {level }}\right\}_{\min E}$

- Case $:$ application =video conferencing, multicasting

$\mathrm{P}=\left\{\text { Paths } / \mathrm{B}_{\text {avai }}>=\mathrm{B}_{\min } \text { and } \mathrm{S}>=\mathrm{S}_{\text {level }}\right\}_{\min }$ (WCETT,E) 
We denote $\mathrm{P}$ as the selected path. Our algorithm adopts different selection criteria based on the application's flow type (voice, video...). For example, for voice the selected route will be the path that minimises energy while having an end-to-end delay least or equal to the max tolerated application delay $T_{\max }$ and a security level superior or equal to the $S_{\text {level }}$ required by the application.

Step 4: Route Activation: The route is activated by the data transmission of the actual traffic flow, and bandwidth reservation will take effect.

The choice of radio technology influences the performance of the network and thus the routing protocol needs to be aware of it, and cannot operate in the same way as wired networks which are agnostic about the underlying medium. For better path selection process, we introduce technologies specificities and preferences in the routing algorithm through the value that we attribute to the link energy consumption parameter $E_{i}$ and link security level parameter $S_{\mathrm{i}}$. for example: $S_{\mathrm{i}}$ is high for an Ethernet link and low for an insecure WiFi link. Respectively, $\mathrm{E}_{\mathrm{i}}$ is high for wireless connections and low for an Ethernet link.

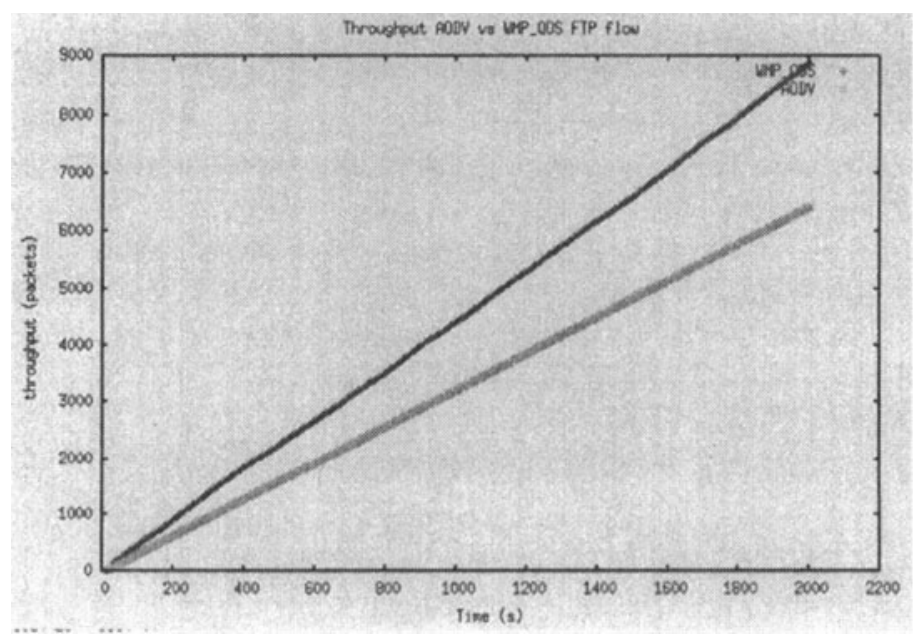

Figure-1: WMP_QoS versus AODV under FTP Flow 


\subsection{Performance results}

We evaluate the performance of WMP_QoS under using the $n s-2$ network simulator [12]. Mainly two types of traffic sources are used (FTP and voice). The source-destination pairs are spread randomly over the network. First, an attempt was made to compare WMP_QoS to the basic AODV standard under the same application flow.

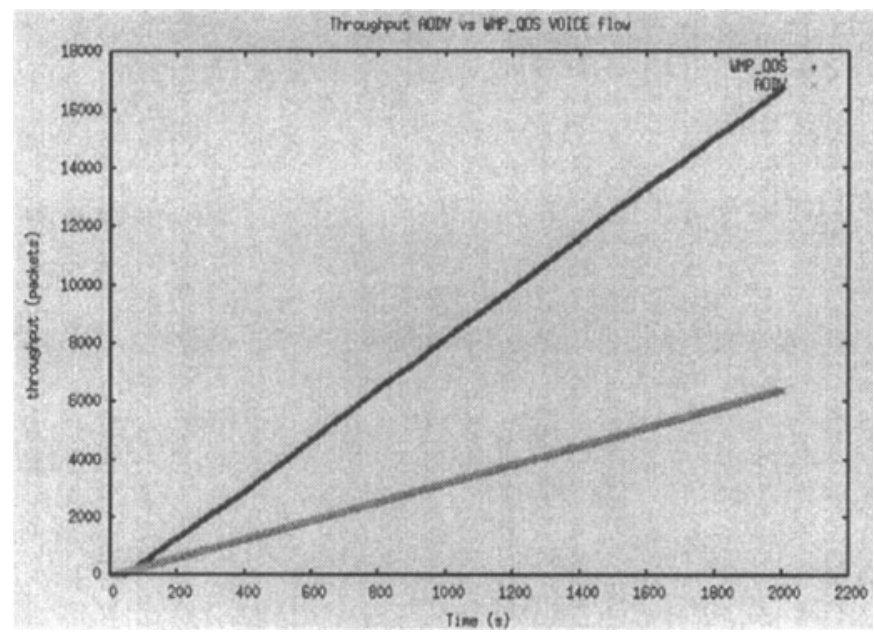

Figure-2: WMP_QoS versus AODV under voice Flow

Figure 1-2 shows that WMP_QoS outperforms AODV under the two types of applications flows. To confirm that WMP_QoS offers a differentiated routing service per application type, we have compared in Figure 3 the end-to-end delay under WMP_QoS for an FTP and voice traffic. The same source and destination nodes where used in both scenarios. You can see that the average end-to-end delay of packet delivery was higher in FTP as compared to voice flow. This means that WMP_QoS choose a different path for FTP and voice flow, while trying to minimize the delay for voice because it's a time sensitive application. 


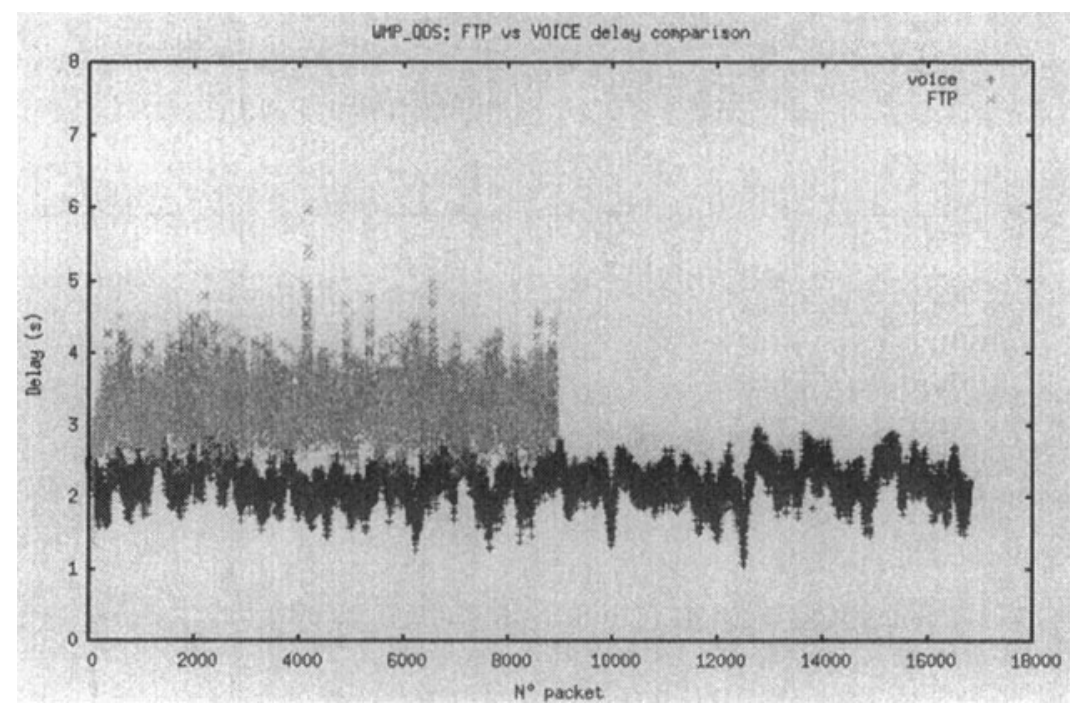

Figure-3: end to end delay FTP vs voice under WMP_QoS

\section{Conclusion}

Based on an analysis of existing routing metrics in wireless mesh networks, we have presented in this paper a QoS routing protocol called WMP_QoS for multi-hop home networks. Our goal is to maintain a stable route which provides per flow guarantee quality of service while taking advantage of heterogeneous link layer characteristics. We have shown through simulations the viability of our protocol.

\section{References}

[1]. Firetide Networks. www.firetide.com

[2]. R. Ogier, F. Templin, M. Lewis, Topology dissemination based on reverse-path forwarding (TBRPF), IETF RFC 3684, February 2004. 
[3]. Microsoft Mesh Networks:http:// research.microsoft.com/mesh

[4]. D.B. Johnson, D.A. Maltz, Y.-C. Hu, The dynamic source routing protocol for mobile ad hoc networks (DSR), IETF Internet-Draft: work in progress, July 2004.

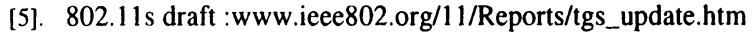

[6]. C. Perkins, E. Belding-Royer, S. Das, Ad hoc on-demand distance vector (AODV) routing, IETF RFC 3561, July 2003.

[7]. Woo, T. Tong, and D. Culler. Taming the underlying challenges of reliable multihop routing in sensor networks. In SenSys, 2003.

[8]. Richard Draves, Jitendra Padhye, and Brian Zill, Comparison of routing metrics for static multi-hop wireless networks. Proceedings of the 2004 conference on Applications, technologies, architectures, and protocols for computer communications, August 2004.

[9]. D.S.J. De Couto, D. Aguayo, J. Bicket, R. Morris, A high-throughput path metric for multi-hop wireless routing, in: ACM Annual International Conference on Mobile Computing and Networking (MOBICOM), 2003, pp. 134-146

[10]. WCETT: R. Draves, J. Padhye, B. Zill, Routing in multi-radio, multi-hop wireless mesh networks, in: ACM Annual International Conference on Mobile Computing and Networking (MOBICOM), 2004, pp. 114-128.

[11]. Akyildiz, I.F., Wang, X. and Wang, W., Wireless Mesh Networks: A Survey, Computer Networks Journal (Elsevier), March 2005.

[12]. NS-2 simulator, www.isi.edu/nsnam/ns/ 